\title{
DESARROLLO INFANTIL TEMPRANO EN EL PERÚ
}

\section{EARLY CHILDHOOD DEVELOPMENT IN PERU}

\author{
Melitón Arce ${ }^{1, a}$
}

\begin{abstract}
El presente número de Revista Peruana de Medicina Experimental y Salud Pública-RPMESP-contiene importantes artículos referidos a la salud del niño en la etapa temprana de su vida. Está dedicado, de una parte, a difundir conocimiento actualizado sobre lo que atañe al crecimiento y desarrollo en esta trascendente edad y, de otra, a algunos aspectos que, en el presente, constituyen temas de interés para contribuir a su mayor bienestar, en el entendido de que más allá de lo que expresa el simple discurso, de su calidad de vida depende el futuro de la sociedad.
\end{abstract}

Haciendo una breve referencia de tales artículos, sin pretender entrar en aspectos de detalle, a los cuales el lector tendrá la oportunidad de acceder directamente, señalaremos lo que en un breve espacio como el presente puede adelantarse de cada uno de ellos, asignándoles lo que a juicio del editor constituye la parte medular de su mensaje.

El primero que comentamos, por constituir sin duda el artículo medular, es el escrito por distinguidos especialistas miembros de la Sociedad Peruana de Pediatría: María del Pilar Medina Alva, Inés Caro Kahn, Pamela Muñoz Huerta, Janette Leyva Sánchez y José Moreno Calixto, con la participación y coordinación de su presidenta la Dra. Sarah María Vega Sánchez, que describe las diversas fases del desarrollo neurológico del niño, incidiendo en el proceso y la cronología de la maduración de cada una de sus principales áreas, así como de los signos de alerta de probables alteraciones, que deben tenerse presentes para buscar su corrección (1). De su lectura se infiere la importancia de atender cabalmente la salud integral del niño, desde la más temprana edad, a efecto de facilitar el pleno despliegue de su potencial genético y, de ese modo, dejarle alcanzar cada meta en el momento previsto, permitiéndole así un desarrollo normal.

Otro de los artículos se refiere a la mortalidad neonatal y describe los resultados de una investigación realizada a nivel nacional por Jeannette Ávila, Mario Tavera y Marco Carrasco, quienes basados en las notificaciones oficiales de los años 2011-2012, aparte de comprobar un elevado índice de subregistro, $52,9 \%$, y una tasa de mortalidad de 12,8 por 1000 nacidos vivos, encuentran entre las causas más frecuentes la inmadurez por prematuridad y la infección, la primera más frecuente en la costa y la segunda en la sierra y selva ${ }^{(2)}$. La tasa referida nos lleva a comentar que, de acuerdo con la tasa actual de mortalidad infantil de 16 por mil, la mortalidad neonatal estaría contribuyendo a ella con los $2 / 3$, relación que, como sabemos, hasta hace algunos años era a la inversa. Otro aspecto relevante es el de enfatizar la necesidad de procurar que el máximo número de nacimientos ocurran a término y, desde luego, seguir promoviendo el parto institucional.

Sobre el mismo tema, Adrián V. Hernández, Vinay Pasupulet, Vicente Benites-Zapata, Enrique Velásquez-Hurtado, Jessica Loyola-Romaní, Yuleika Rodríguez-Calviño, Henry Cabrera Arredondo, Marco Gonzales-Noriega y Walter Vigo-Valdez, reportan los resultados de una evaluación sobre costoefectividad de cinco intervenciones en la comunidad, orientadas a reducir tal mortalidad, encontrando como efectivas solo a dos de ellas: la educación sanitaria de las madres y el cuidado de los niños en casa de madres. Las demás, como suplementación materna con multimicronutrientes, lo propio con vitamina A, e incluso, la estrategia Madre Canguro, no mostraron efectividad ${ }^{(3)}$.

\footnotetext{
1 Academia Nacional de Medicina. Lima, Perú

a Editor invitado

Recibido: : 16-09-15 Aprobado: 16-09-15
}

Citar como: Arce M. Desarrollo infantil temprano en Perú [editorial]. Rev Peru Med Exp Salud Publica. 2015;32(3):419-20. 
Es interesante también, el estudio realizado por Gandy Dolores-Maldonado, Doris Delgado-Pérez y Hilary Creed-Kanashiro, que revisa las diferentes acciones que las madres emplean para hacer atractivo el acto de comer de sus hijos, encontrando que todas ellas resultan eficaces y que son las madres multíparas las que demuestran mayor habilidad y eficiencia, no así las primíparas que asumen una actitud más autoritaria. Sin duda, esto último debe considerarse como consecuencia de su relativa inexperiencia ${ }^{(4)}$.

Otra comunicación realizada por investigadores del Instituto de Salud del Niño, Carlos Del Águila, Juan Falen-Boggio y Oscar Espinoza y de la Universidad de Kansas, Lina Huerta-Sáenz y Naim Mitre, se aborda el tema del hipotiroidismo congénito y, si bien encuentra, comparando dos estudios previos, que la prevalencia entre 1984, entonces de 1:1250, se había reducido el 2007 a 1:1638, una evaluación sobre la edad promedio de diagnóstico efectuada en el Instituto, de los casos observados entre 1995 y 2005 , demuestra que esta se realiza a una edad promedio de 5,9 meses, momento en que el daño que este defecto ocasiona al niño resulta es ya irreparable. Concluyen afirmando la ineficiencia actual en su detección en nuestro medio e invocando una aplicación más efectiva del tamizaje respectivo ${ }^{(5)}$.

La hepatitis A, cuya vacuna fue descubierta en 1986 sigue interesando a nuestros investigadores en lo referente a su incidencia y prevalencia, dados los riesgos que la infección plantea. Respecto a ella, Roger Hernández, Eduardo Chaparro, Carlos Díaz, Martha Carbajal, Érico Cieza y Rosario Cerpa, reportan un estudio sobre la presencia de anticuerpos en el suero de 1721 niños y adolescentes de cinco ciudades del país, encontrándola en el 50,6\% de ellos, siendo esta mayor en lquitos, $68,7 \%$, y menor en Lima, 37,4\%, y mayor también entre los de mayor edad y en los pertenecientes a los estratos socioeconómicos de menores recursos ${ }^{(6)}$. Si bien estos resultados pueden, en alguna medida, ser reflejo de la etapa previa a la inclusión de la vacuna en el programa, no es menos cierto que debe afianzarse su uso y aplicarse en forma universal.

Finalmente, no podía faltar en este número el tema de la anemia, cuya prevalencia entre nuestros niños sigue siendo alta y que, como sabemos, tiene profunda repercusión negativa en su desarrollo neurointegrativo. Sobre esta afección, Elena Gonzales, Lucio HuamánEspino, César Gutiérrez, Juan Pablo Aparco y Jenny Pillaca, estudian su prevalencia en Huancavelica y en Coronel Portillo, realizando determinaciones séricas de ferritina, vitamina B12 y ácido fólico intraeritrocitario, las que complementan con examen parasitológico en los detectados con anemia. Reportan una prevalencia de $55,9 \%$ en Huancavelica y de $36,2 \%$ en Coronel Portillo y como causa asociada más frecuente en ambas a la parasitosis, recomendando que en causalidad de la anemia deban considerarse factores diferentes a la simple carencia de hierro ${ }^{(7)}$.

Concluimos esta nota editorial resaltando la importancia que el cuidado de la salud del niño en la etapa temprana de su vida es fundamental no solo para que crezca y desarrolle plenamente de acuerdo al potencial genético que porta, sino también para alcanzar una adultez que le permita contribuir a la creciente prosperidad de su entorno familiar y social y ulteriormente a la construcción de comunidades cada vez mejores.

\section{REFERENCIAS BIBLIOGRÁFICAS}

1. Medina Alva MDP, Caro Kahn I, Muñoz Huerta P, Leyva Sánchez J, Moreno Calixto J, Vega Sánchez SM. Neurodesarrollo infantil: características normales y signos de alarma en el niño menor de cinco años. Rev Peru Med Exp Salud Publica. 2015;32(3):565-73.

2. Ávila J, Tavera M, Carrasco M. Características epidemiológicas de la mortalidad neonatal en el Perú, 20112012. Rev Peru Med Exp Salud Publica. 2015;32(3):423-30.

3. Hernández AV, Pasupuleti V, Benites-Zapata V, Velásquez-Hurtado E, Loyola-Romaní J, Rodríguez-Calviño $\mathrm{Y}$, Cabrera-Arredondo H, Gonza-
les-Noriega M, Vigo-Valdez W. Revisión sistemática sobre la efectividad de intervenciones basadas en la comunidad en la disminución de la mortalidad neonatal. Rev Peru Med Exp Salud Publica. 2015;32(3):532-45.

4. Dolores-Maldonado G, Delgado-Pérez $\mathrm{D}$, Creed-Kanashiro $\mathrm{H}$. Estrategias de la madre durante el proceso de alimentación en niños de 6 a 24 meses de dos comunidades rurales de Lima, Perú. Rev Peru Med Exp Salud Publica. 2015;32(3):440-8.

5. Huerta-Sáenz L, Del Águila C, Espinoza O, Falen-Boggio J, Mitre N. Tamizaje nacional unificado de hipotiroidismo congénito en el Perú: un programa inexistente. Rev Peru Med Exp Salud Publica. 2015;32(3):574-8.

6. Hernández R, Chaparro E, Díaz C, Carbajal M, Cieza E, Cerpa R. Frecuencia de hepatitis A en niños y adolescentes de cinco ciudades del Perú. Rev Peru Med Exp Salud Publica. 2015;32(3):492-8.

7. Gonzales E, Huamán-Espino L, Gutiérrez C, Aparco JP, Pillaca J. Caracterización de la anemia en niños menores de cinco años de zonas urbanas de Huancavelica y Ucayali en Perú. Rev Peru Med Exp Salud Publica. 2015;32(3):431-9. 